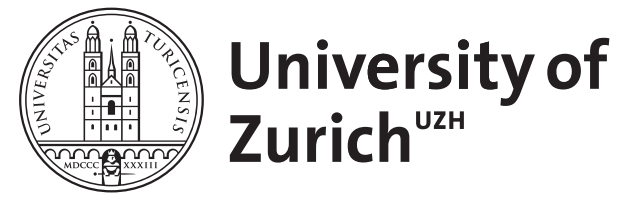

\title{
Kongenitale Nävi im Kindesalter
}

Wälchli, R ; Theiler, M ; Neuhaus, K ; Weibel, L

\begin{abstract}
Nevi represent congenital hamartomatous malformations of various components of the skin. The most common forms are congenital melanocytic nevi (CMN) and nevi of epithelial origin (epidermal and organoid nevi). Large CMN in particular can lead to severe complications and the management of those affected represents a challenge from birth. In contrast to previous assumptions, the risk of malignancy from CMN is considered to be relatively low however, this may be relevantly increased in certain situations. Possible extracutaneous symptoms in cases of central nervous system (CNS) involvement should not be underestimated and early imaging investigations are part of the routine diagnostic procedure. Surgical measures are still very important in the treatment of CMN but the indications must be weighed up for each individual case. Patients often experience marked stigmatization due to disfigurement by their birthmark and this needs to be taken into consideration for their treatment. The most common epithelial nevi are sebaceous nevi. In 2-13\% of cases additional tumors occur within this nevus and early surgical excision is indicated in most cases, not least for aesthetic reasons. If generalized spreading of epidermal nevi occurs, additional investigations are necessary to exclude associated ophthalmological, cardiac or neurological malformations.
\end{abstract}

DOI: https://doi.org/10.1007/s00105-012-2410-3

Other titles: Congenital nevi in childhood

Posted at the Zurich Open Repository and Archive, University of Zurich

ZORA URL: https://doi.org/10.5167/uzh-91298

Journal Article

Published Version

Originally published at:

Wälchli, R; Theiler, M; Neuhaus, K; Weibel, L (2013). Kongenitale Nävi im Kindesalter. Der Hautarzt, 64(1):32-39.

DOI: https://doi.org/10.1007/s00105-012-2410-3 
Hautarzt 2013 • 64:32-39

DOI 10.1007/s00105-012-2410-3

Online publiziert: 21. Januar 2013

(c) Springer-Verlag Berlin Heidelberg 2013

R. Wälchli ${ }^{1} \cdot$ M. Theiler $^{1,2} \cdot$ K. Neuhaus $^{3} \cdot$ L. Weibel ${ }^{1,2,4}$

${ }^{1}$ Abteilung für Pädiatrische Dermatologie, Kinderspital Zürich

${ }^{2}$ Dermatologische Klinik, Universitätsspital Zürich

${ }^{3}$ Abteilung für Plastische Chirurgie, Kinderspital Zürich

${ }^{4}$ Leitende Ärztin Dermatologie, FMH Dermatologie/Venerologie und FMH Kinder- und Jugendheilkunde; Kinderspital Zürich

\section{Kongenitale Nävi im Kindesalter}

ferzellen, die bereits intrauterin entstehen (1. Trimester) und bei Geburt oder seltener innerhalb der ersten Lebensmonate sichtbar sind. Klinisch handelt es sich um heterogene hell- bis dunkelbraun pigmentierte Makulae oder Plaques unterschiedlicher Größe, die eine homogene oder inhomogene Oberflächenstruktur und oft eine verstärkte Behaarung (Hypertrichose) aufweisen. Insbesondere bei großen CMN finden sich gelegentlich dunkelbraune oder hautfarbenen Knoten innerhalb des Nävus; 75\% der großen CMN $(>20 \mathrm{~cm})$ werden von zahlreichen kleineren sog. Satellitennävi begleitet, die bereits bei Geburt oder in den ersten 5 Lebensjahren auftreten.

Die Häufigkeit von CMN jeglicher Größe beträgt 1-2\%, während $\mathrm{CMN}$ mit einer Fläche größer als $20 \mathrm{~cm}^{2}$ selten sind (Inzidenz 1:500.000; [1]).

Text ist eine Zusammenfassung des Referates „Kongenitale Nävi“ von Frau Dr. Lisa Weibel während den Zürcher Dermatologischen Fortbildungstagen 2012 und entspricht nicht einem herkömmlichen Review-Artikel. Der Beitrag beschreibt das heutige Vorgehen am UniversitätsKinderspital Zürich unter Hervorhebung praktischer Hinweise im Management dieser z. T. komplexen Patienten.

\section{Kongenitale melanozytäre Nävi}

\section{Epidemiologie, Klassifikation und Klinik}

CMN sind gutartige Proliferationen von Melanozyten oder Melanozytenvorläu-

\section{\) Die Häufigkeit von kongenitalen melanozytären Nävi beträgt 1-2\%}

Gemäß neu vorgeschlagener Klassifikation werden $C M N$ in kleine $(<1,5 \mathrm{~cm})$, mittelgroße (M1: 1,5-10 cm, M2:>10-20 cm), große (L1: $20-30 \mathrm{~cm}, \mathrm{~L} 2: 30-40 \mathrm{~cm}$ ), riesige (G1: 40-60 cm, G2 >60 cm) Nävi und multiple mittelgroße CMN ( $\geq 3$ Stück) unterteilt, wobei sich die Zahlen auf die prognostizierte Größe im Erwachsenenalter beziehen [2]. Letztere kann im Säuglingsalter anhand eines Faktors ermit- telt werden (Kopf xl,7, Hals/Stamm/Arme/Füße x2,8, Beine x3,3; [3]). Die neue Klassifikation von CMN berücksichtigt neben der Größe auch die Lokalisation (Kopf, Stamm, Extremitäten), die Satellitenzahl ( $\mathrm{S} 0-\mathrm{S} 3=0$ bis $>50$ Satelliten) und die Morphologie (Heterogenität, unregelmäßige Oberfläche, Knoten und Behaarung) der CMN [2]. Anhand dieser Faktoren können das Risiko für Komplikationen abgeschätzt und entsprechende Maßnahmen veranlasst werden (s. unten).

\section{Pathogenese und genetische Aspekte}

CMN bestehen aus einer Akkumulation von melanozytären Zellen, die von der Neuralleiste abstammen. Während der Embryogenese wandern diese Melanoblasten aus der Neurralleiste entlang der Leptomeningen in die fetale Dermis ein und von dort in die Epidermis, wo sie sich zu reifen Melanozyten ausdifferenzieren. Während dieser Migration können Fehlregulationen auftreten, die zu einer unkontrollierten Proliferation dieser Zellen führen. Je nach Zeitpunkt und Lokalisation resultieren unterschiedliche klinische Erscheinungsformen an verschiedenen Organen (• Abb. 1). Die melanozytären Vorläuferzellen weisen zudem das Potenzial für invasives und proliferatives Wachstum auf.

Kommt es zusätzlich zu einer Nävuszellaussaat im ZNS, so äußert sich dies klinisch in Form der neurokutanen Melanozytose (Pigmentzellansammlungen lepto- 


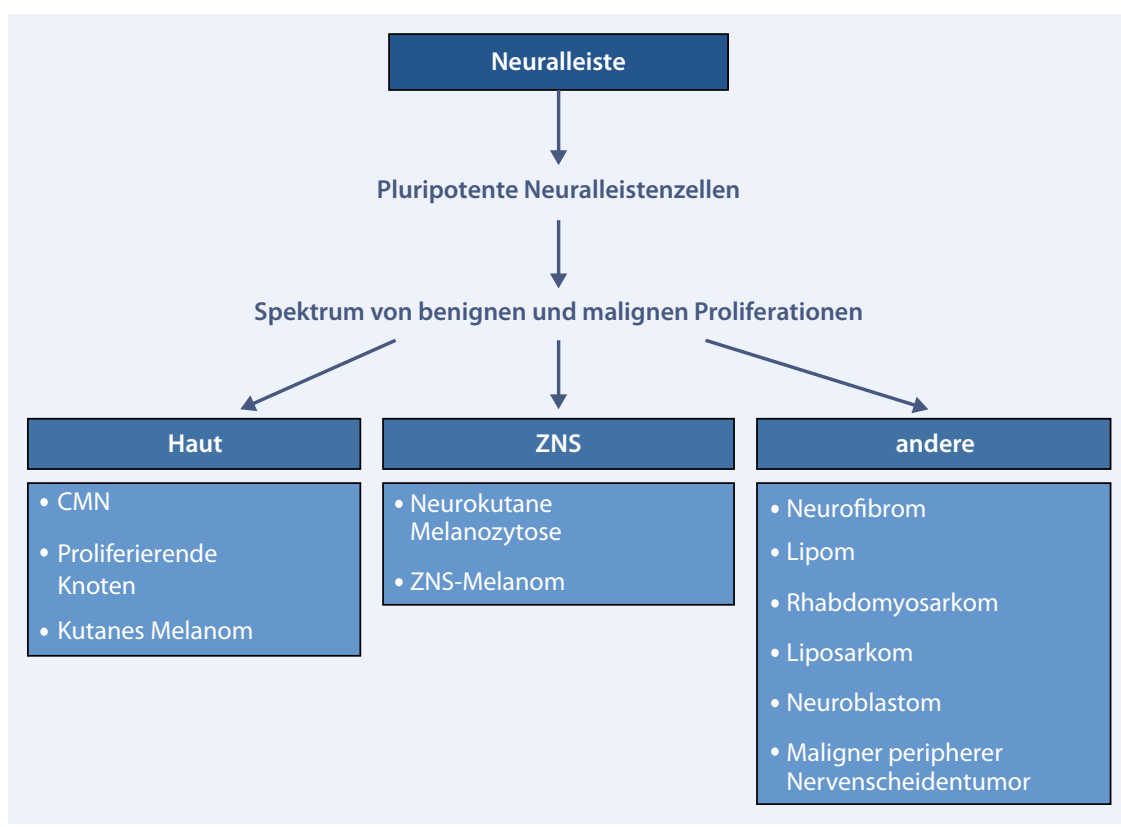

Abb. $1 \Delta$ Entstehung von kongenitalen melanozytären Nävi (CMN) und CMN-assoziierten Tumoren. (Adaptiert nach [4])

meningeal inklusive Spinalkanal oder intrazerebral). Auch können sich aus diesen pluripotenten Vorläuferzellen andere, sowohl benigne als auch maligne Tumoren, wie z. B. Neurofibrome, Lipome, Liposarkome, Rhabdomyosarkome und Neuroblastome, entwickeln (• Abb. 1, [4]).

Bis heute ist insgesamt nur wenig über die Ätiologie von CMN bekannt. Die molekulare Tumorbiologie von CMN zeigt im Gegensatz zum Melanom selten chromosomale Anomalien. Bezüglich der Mediatoren des MAP-Kinase-Signalwegs findet sich Folgendes: Große CMN und proliferierende Knoten weisen häufig (70$80 \%$ ) NRAS-Mutationen auf, jedoch keine BRAF-Mutationen; bei kleinen CMN hingegen finden sich - gleich wie bei erworbenen melanozytären Nävi - BRAF-Mutationen häufig [5, 6]. Kürzlich konnte gezeigt werden, dass große CMN in Analogie zum Melanom SOX10 exprimieren [7]. SOX10 ist ein zentraler Transkriptionsfaktor, der für die Ausbildung von Melanozyten aus der Neuralleiste verantwortlich ist und somit auch ein potenzielles Ziel für zukünftige zielgerichtete Therapien darstellen könnte. Ein neuer Aspekt ist die Diskussion einer zugrunde liegenden genetischen Prädisposition. Neuere Untersuchungen von Kinsler et al. [8] ergaben, dass $25 \%$ der betroffenen Patienten eine positive Familiengeschichte für CMN ha- ben und Kinder mit CMN gemeinsame phänotypische Merkmale im Sinne einer charakteristischen Fazies aufweisen. Des Weiteren findet sich bei Kindern mit großen CMN eine signifikante Häufung bestimmter Melanocortin-1-Rezeptor-Varianten, einhergehend mit einem erhöhten Anteil des rothaarigen und sommersprossigen Phänotyps [9]. Diese Erkenntnisse ließen Kinsler den Begriff des „congenital melanocytic naevi syndrome "postulieren.

\section{Komplikationen und Aspekte für das klinische Management}

In der Folge werden die wichtigsten Aspekte für das Management von Patienten mit CMN diskutiert:

- Entartungsrisiko,

- ZNS-Beteiligung,

- Therapie und psychosoziale Aspekte.

\section{Entartungsrisiko von kongenitalen melanozytären Nävi}

Das Risiko der Entstehung eines malignen Melanoms auf dem Boden von CMN ist insgesamt deutlich geringer als früher angenommen und korreliert in erster Linie mit der Nävusgröße. Bei der Diskussion des Melanomrisikos von CMN gilt es prinzipiell, zwischen dem absoluten Risiko (=lebenslang) und relativen Risiko (=im Quervergleich zur korrelieren- den Altersgruppe) zu unterscheiden. Bei großen und riesigen Nävi $(>20 \mathrm{~cm})$ beträgt das absolute Melanomrisiko 1-10\%; bei mittelgroßen Nävi um $1 \%$ und bei kleinen Nävi um 1\%o $[10,11,12]$.

Die relativen Risiken einer Melanomentwicklung liegen deutlich höher. Es gilt zudem zu beachten, dass ca. 30\% der malignen kutanen Melanome bei Patienten mit großen oder Riesennävi nicht innerhalb des Nävus, sondern anderswo am Körper auftreten [11]. Bei den großen CMN entwickeln sich die Melanome in der Regel unterhalb der dermoepidermalen Junktionszone. Sie sind daher klinisch und dermatoskopisch nicht sichtbar und werden oft erst spät erkannt.

D Neben der Inspektion stellt die Palpation einen integralen Bestandteil der ärztlichen Untersuchung dar.

Mehr als 70\% der kindlichen Melanome, die auf dem Boden eines großen CMN entstehen, manifestieren sich innerhalb der ersten Lebensjahre. Beide Umstände werden mit dem Vorliegen unreifer genetisch instabiler melanozytärer Vorläuferzellen in tieferen Dermisschichten erklärt. Im Gegensatz dazu entstehen Melanome in kleinen und mittelgroßen CMN meist erst im Erwachsenenalter und im Bereich der dermoepidermalen Junktionszone und sind somit klinisch besser zugänglich.

Folgende Risikofaktoren sind heute für die Entstehung eines kutanen Melanoms bei CMN bekannt [10, 11, 12]:

- große $(>20 \mathrm{~cm})$ und insbesondere riesige Nävi $(>40 \mathrm{~cm})$,

- Lokalisation des CMN am Stamm,

— inhomogene Nävi mit zahlreichen proliferierenden Knoten.

Multiple Satellitenläsionen hingegen stellen keinen Risikofaktor einer malignen Entartung dar und zeigen selbst auch keine Entartungstendenz.

\section{ZNS-Beteiligung}

Neben der malignen Entartung stellt eine mögliche ZNS-Beteiligung bei großen und riesigen CMN ein Morbiditäts- respektive Mortalitätsrisiko dar. Diese wird bei ca. 10-20\% der Patienten (mit Nävusgröße $>20 \mathrm{~cm}$ ) gesehen entweder in Form einer neurokutanen Melanozytose (lepto- 
meningeale oder intrazerebrale Melanose) oder in Form von ZNS-Fehlbildungen (Malformationen, Hydrozephalus) oder nichtmelanozytären ZNS-Tumoren $[4,13]$. Folgende Risikofaktoren sind heute für eine ZNS-Beteiligung bei CMN bekannt $[13,14]$ :

- $\mathrm{CMN}>20 \mathrm{~cm}$,

- multiple Satelliten (>20 Stück),

- mehrere mittelgroße CMN.

Entgegen früheren Annahmen stellt hierbei eine Lokalisation über der posterioren Körperachse keinen Risikofaktor dar [10]. Bei Vorliegen oben genannter Risikofaktoren ist die Durchführung einer Magnetresonanztomographie (MRT) des Schädels (und Rückenmarks) bis spätestens im Alter von 6 Monaten zu empfehlen (zu einem späteren Zeitpunkt ist diese Untersuchung bei abgeschlossener Myelinisierung des ZNS nicht mehr sensitiv genug). Bei Vorliegen einer ZNS-Fehlbildung oder eines Tumors können evtl. neurochirurgische Interventionen notwendig werden.

\section{》) Eine symptomatische neurokutane Melanozytose hat eine schlechte Prognose}

Die klinische Bedeutung und Prognose einer neurokutanen Melanozytose ist vom Vorhandensein oder Fehlen neurologischer Symptome abhängig, wobei diese in der Regel innerhalb der ersten 2 Lebensjahre auftreten. Eine symptomatische neurokutane Melanozytose (Hydrozephalus, Epilepsie, Hirndruckzeichen, Rückenmarkkompression) hat prinzipiell eine schlechte Prognose und verläuft in $35-50 \%$ der Fälle letal. Bei lediglich radiologisch nachweisbarer neurokutaner Melanozytose und klinisch-neurologisch unauffälligem Kind ist die Prognose hingegen eher günstig $[13,14]$. Die $\bullet$ Abb. 2 beinhaltet das an unserem Zentrum angewendete Schema zur Diagnostik von ZNS-Komplikationen bei CMN und deren Management.

\section{Therapie und psychosoziale Aspekte}

Bis heute ist nicht klar, ob die Exzision eines großen/Riesennävus das Risiko der

Hautarzt 2013 - 64:32-39 DOI 10.1007/s00105-012-2410-3

๑) Springer-Verlag Berlin Heidelberg 2013

\section{R. Wälchli $\cdot$ M. Theiler $\cdot$ K. Neuhaus $\cdot$ L. Weibel Kongenitale Nävi im Kindesalter}

\section{Zusammenfassung}

Nävi stellen kongenitale hamartomatöse Fehlbildungen unterschiedlicher Bestandteile der Haut dar. Am häufigsten treten kongenitale melanozytäre Nävi (CMN) auf, gefolgt von Nävi epithelialen Ursprungs (epidermale und organoide Nävi). Insbesondere große CMN können zu schwerwiegenden Komplikationen führen, und das Management der Betroffenen stellt ab Geburt eine Herausforderung dar. Entgegen früheren Annahmen ist das Risiko einer malignen Entartung von CMN insgesamt als eher gering anzusehen, steigt jedoch in speziellen Situationen relevant an. Nicht zu vernachlässigen sind mögliche extrakutane Symptome im Falle einer ZNS-Beteiligung, und frühe bildgebende Untersuchungen gehören heute zur Routinediagnostik. Chirurgische Maßnahmen haben noch immer einen hohen Stellenwert in der Behandlung von CMN, die Indikation da- zu ist jedoch von Fall zu Fall individuell abzuwägen. Nicht zuletzt leiden die Patienten häufig stark an der ästhetischen Beeinträchtigung durch ihr Muttermal, sodass in der Behandlung auch diesem Punkt Rechnung getragen werden muss. Der Naevus sebaceus ist der häufigste Vertreter der epithelialen Nävi. In 2-13\% treten darin Zusatztumoren auf, und eine frühe chirurgische Exzision ist in den meisten Fällen nicht zuletzt auch aus ästhetischen Überlegungen sinnvoll. Epidermale Nävi erfordern bei generalisierter Ausdehnung Zusatzuntersuchungen zum Ausschluss von assoziierten ophthalmologischen, kardialen oder neurologischen Fehlbildungen.

\section{Schlüsselwörter}

Kongenitale melanozytäre Nävi · Naevus Ota . Naevus spilus · Nävoide Hypermelanose . Naevus sebaceus

\section{Congenital nevi in childhood}

\section{Abstract}

Nevi represent congenital hamartomatous malformations of various components of the skin. The most common forms are congenital melanocytic nevi (CMN) and nevi of epithelial origin (epidermal and organoid nevi). Large CMN in particular can lead to severe complications and the management of those affected represents a challenge from birth. In contrast to previous assumptions, the risk of malignancy from CMN is considered to be relatively low however, this may be relevantly increased in certain situations. Possible extracutaneous symptoms in cases of central nervous system (CNS) involvement should not be underestimated and early imaging investigations are part of the routine diagnostic procedure. Surgical measures are still very important in the treatment of CMN but the indications must be weighed up for each indi- vidual case. Patients often experience marked stigmatization due to disfigurement by their birthmark and this needs to be taken into consideration for their treatment. The most common epithelial nevi are sebaceous nevi. In 2-13\% of cases additional tumors occur within this nevus and early surgical excision is indicated in most cases, not least for aesthetic reasons. If generalized spreading of epidermal nevi occurs, additional investigations are necessary to exclude associated ophthalmological, cardiac or neurological malformations.

\section{Keywords}

Congenital melanocytic nevi - Nevus of Ota . Nevus, pigmented $\cdot$ Nevoid hypermelanosis . Nevus sebaceous
Entstehung eines Melanoms beim Patienten insgesamt signifikant vermindert oder eliminiert. Von einer gewissen Risikoreduktion durch Exzision wird jedoch generell ausgegangen. Gemäß Kinsler et al. [15] kann das Entartungsrisiko bei großen CMN durch eine chirurgische Exzision nur teilweise reduziert werden, da trotz Entfernung des gesamten Nävus stets einige melanozytären Zellen des CMN übrig bleiben. Die an gewissen Zentren prak- tizierte frühe Dermabrasion oder Kürettage ist in diesem Licht kritisch zu sehen, insbesondere da auch häufig eine Repigmentierung nach Intervention aus tieferen Dermisschichten erfolgt. Es gilt zudem zu beachten, dass jegliche Intervention am Nävus nicht zu einer Reduktion der Häufigkeit extrakutan entstehender Melanome führt.

Grundsätzlich ist gemäß diesen Erkenntnissen die einzig zwingende Indi- 


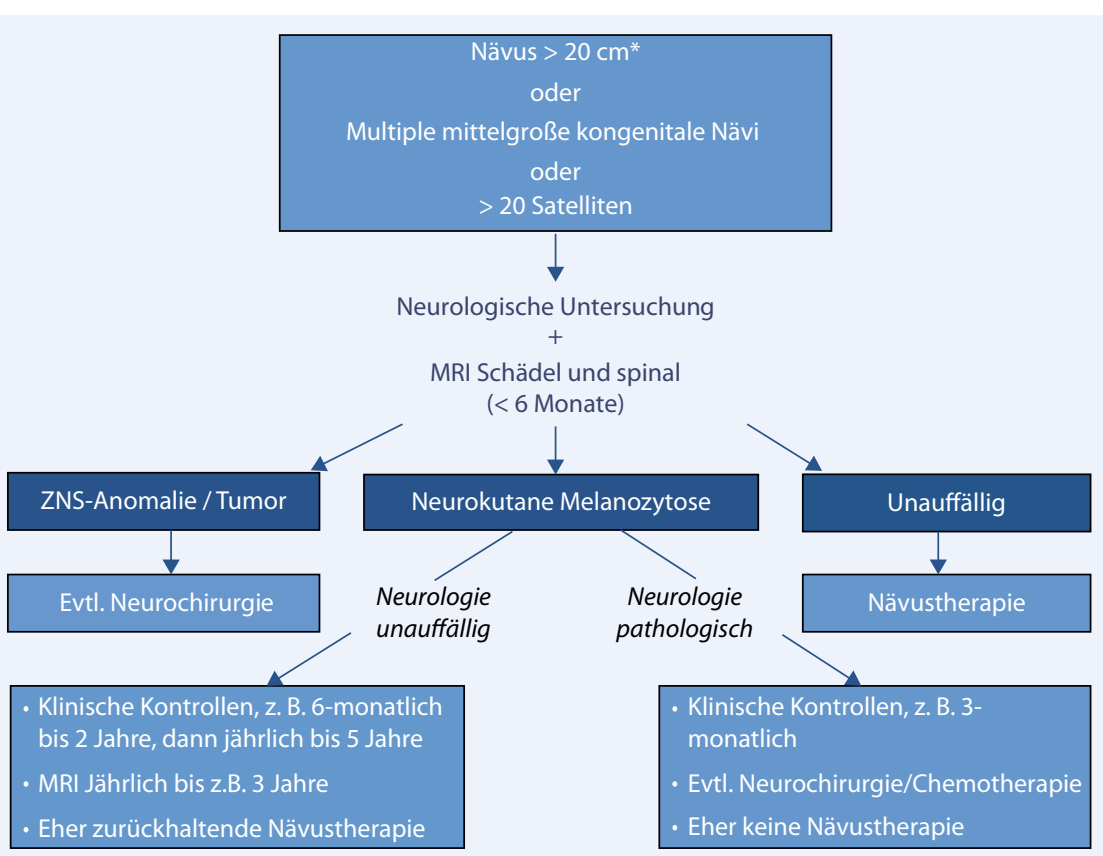

Abb. $2 \Delta$ Am Zürcher Zentrum angewendetes Schema zur Diagnostik von ZNS-Komplikationen und deren Management bei kongenitalen melanozytären Nävi (CMN). * prädiktive Nävus-Erwachsenengröße
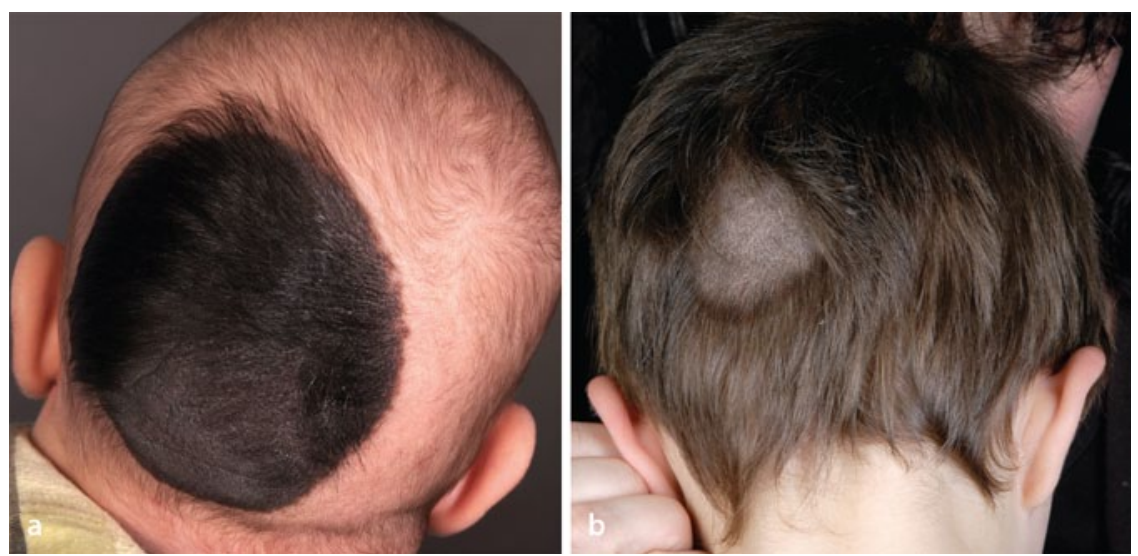

Abb. $3 \Delta$ a Großer, stark behaarter kongenitaler melanozytärer Nävus (CMN) der Kopfhaut bei einem Säugling. b Deutliche spontane Aufhellung des CMN und Rückbildung der Hypertrichose beim gleichen Kind im Alter von 3 Jahren

kation für eine chirurgische Exzision der Verdacht auf Malignität. Selbstverständlich können andere Gründe zu einer chirurgischen Therapie führen, hier steht insbesondere das ästhetische Erscheinungsbild im Vordergrund.

Der Entscheid zu einer Exzision sowie der geeignete Zeitpunkt sind abhängig von verschiedenen Faktoren:

- Einschätzung des absoluten Entartungsrisikos,

- Patienten-/Elternwunsch,

- zu erwartendes Stigmatisierungspotenzial und psychosoziale Belastung, ten mit großem CMN eine Narbe gegenüber dem Nävus bevorzugen, aber etwa ein Viertel der Patienten eine stattgefundene Operation bereuen. Bei CMN der behaarten Kopfhaut kann primär gut abgewartet werden, da diese Nävi oft im Verlauf der ersten Lebensjahre deutlich aufhellen (• Abb. 3, [15]).

\section{》) Die einzige zwingende Indikation für eine chirurgische Exzision ist der Verdacht auf Malignität}

Entscheidet man sich bei einem großen respektive Riesennävus zu einer chirurgischen Intervention, ist ein eher frühes Vorgehen ( $a b$ dem Alter von 12 bis 24 Monaten) sinnvoll, da die chirurgischen Möglichkeiten aufgrund der erhöhten Hautelastizität besser sind und wie erwähnt Melanome in diesen $\mathrm{CMN}$ früh auftreten können. Bei kleinen und mittelgroßen Nävi ist es prinzipiell legitim zuzuwarten, bis der Patient ein Alter erreicht, wo er selbst über eine Intervention entscheiden kann. In folgenden Situationen empfehlen wir an unserem Zentrum jedoch eher eine Exzision:

- dicke, inhomogene, knotige CMN

(• Abb. 4),

- versteckte/schwierig kontrollierbare Lokalisation (• Abb.4),

- hohes Stigmatisierungspotenzial (z. B. starke Behaarung; • Abb. 5).

Werden CMN belasse, sind selbstverständlich regelmäßige, lebenslange dermatologische Verlaufskontrollen indiziert, bei großen Läsionen ab Geburt, bei kleinen bis mittelgroßen ab der Pubertät. Dies kann mit ein Grund sein für den Entscheid, kleine oder mittelgroße Nävi zu exzidieren.

CMN im Gesicht erhebliche Stigmatisierung und Einschränkungen ihrer Lebensqualität erfahren, insbesondere bei großen Nävi und im späteren Schulalter [16, 17]. Dennoch ist das zu erwartende ästhetische Resultat nach operativer Intervention, in der Regel eine oder mehrere Narben, kritisch gegenüber dem Nävus abzuschätzen. Diesbezüglich beobachteten Marghoob et al. ([10] und Marghoob AA, nicht veröffentlicht), dass 76\% der Patien-

\section{Weitere kongenitale Pigmentanomalien}

\section{Naevus spilus}

Der Naevus spilus („speckled lentiginous nevus") ist gekennzeichnet durch einen Café-au-lait-Fleck, auf dem sich im Kindesalter zahlreiche dunklere, makulöse 


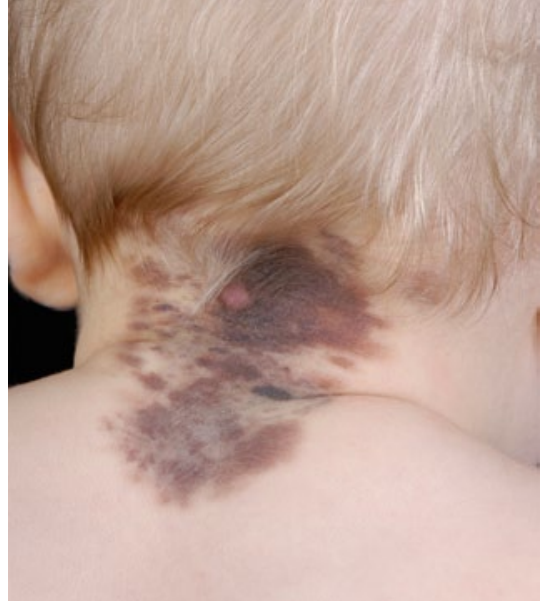

Abb. $4 \Delta$ Inhomogener, mit Knoten besetzter kongenitaler melanozytärer Nävus an schwierig kontrollierbarer Lokalisation. Eine chirurgische Therapie (Totalexzision) ist hier eher zu empfehlen, idealerweise im Kleinkindesalter

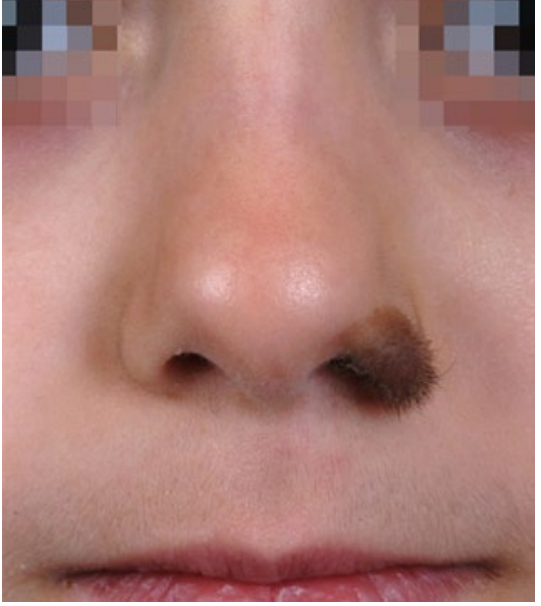

Abb. $5 \Delta$ Kleiner kongenitaler melanozytärer Nävus mit hohem Stigmatisierungspotenzial. Eine chirurgische Therapie ist bei entsprechendem Patientenwunsch sinnvoll

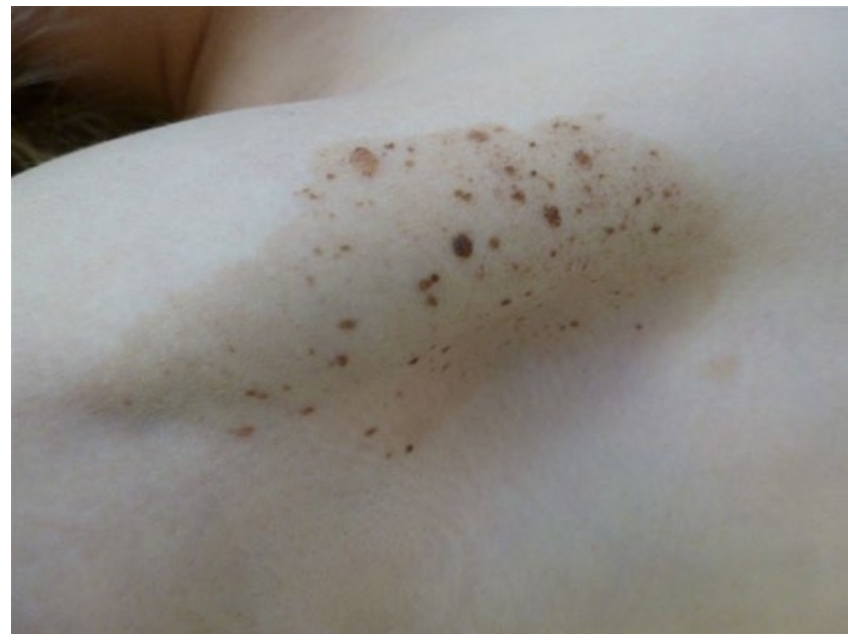

Abb. $6<$ Naevus spilus (,speckled lentiginous nevus") an der Schulter oder kleinpapulöse Einsprengsel ausbilden (• Abb. 6). Letztere entsprechen histologisch CMN, nicht selten liegen jedoch auch spitzoide Läsionen vor [18]. Die Größe des Nävus ist sehr variabel. Der Naevus spilus wird heute von vielen Experten als Sonderform eines CMN angesehen [18, 19]. Daher ist anzunehmen, dass das Entartungsrisiko (Entwicklung eines Melanoms) beim Naevus spilus vergleichbar ist mit dem Entartungsrisiko von klassischen CMN gleicher Größe. Eine prophylaktische Exzision ist im Regelfall nicht erforderlich. Regelmäßige klinische Verlaufskontrollen sind aber indiziert, und bei Malignitätsverdacht sollte eine vollständige Exzision durchgeführt werden.

\section{Naevus Ota}

Der Naevus Ota (Naevus fuscocaeruleus ophthalmicomaxillaris) ist eine Sonderform eines CMN mit Ansammlung der Melanozyten in der Dermis. Zumeist wird er bei Patientinnen asiatischer oder seltener afrikanischer Herkunft gesehen. Klinisch erscheint er als homogene oder fleckige schwarzbläuliche Pigmentierung im Bereich des ersten und/oder zweiten Trigeminusasts, häufig unter Einbeziehung von Konjunktiva und Retina [20]. Die blaue Farbe ist auf den sog. Tyndall-Effekt (Farbänderung des Melanins je nach Tiefe) zurückzuführen. In $60 \%$ der Fälle ist eine Augenbeteiligung assoziiert [21]. Daraus kann sich als Komplikation ein Glau- kom oder ein okuläres Melanom (choroidal, Iris) manifestieren. Daher sind regelmäßige ophthalmologische Untersuchungen indiziert. Das Risiko einer malignen Entartung scheint sich im Bereich von klassischen CMN vergleichbarer Größe zu bewegen.

\section{Nävoide Hypermelanose}

Die nävoide Hypermelanose (Synonym: „linear and whorled naevoid hypermelanosis") ist eine angeborene Pigmentanomalie, die sich bereits bei Geburt oder im frühen Säuglingsalter durch lineare, geschwungene lentiginöse Hyperpigmentierungen entlang den Blaschko-Linien (embryonale Wanderungslinien der Melanozyten) oder als Schachbrettmuster angeordnet präsentiert (• Abb. 7). Histologisch zeigt sich eine epidermale Melanose. Diese Manifestation entspricht einem kutanen Mosaik [22]. Es sind assoziierte Erkrankungen möglich, die z. B. das Nervensystem (Krampfleiden, mentale Retardierung), das muskuloskelettale System (Skelettanomalien) die Augen oder das Herz (angeborene Herzfehler) betreffen [22, 23]. Die Prävalenz dieser assoziierten extrakutanen Anomalien wird mit 5-30\% beschrieben, wir gehen jedoch davon aus, dass dies deutlich seltener auftritt und die große Mehrheit der Patienten eine rein kutane Manifestation aufweist. Bei Kindern ohne klinische Auffälligkeiten und mit einer normalen altersentsprechenden psychomotorischen Entwicklung führen wir somit keine weiteren spezifischen Abklärungen (neuropädiatrische klinische Untersuchung oder Bildgebung) durch. Wir empfehlen, die kinderärztliche Routine-Entwicklungskontrollen sorgfältig durchzuführen und bei Auftreten von neurologischen oder psychomotorischen Auffälligkeiten weiterführende Abklärungen (Bildgebung) einzuleiten. Eine ophthalmologische Untersuchung führen wir bei allen Kindern mit ausgedehnter nävoider Hypermelanose durch, da es sich um eine nichtinvasive Untersuchung mit potenziellen therapeutischen Konsequenzen handelt. Die nävoide Hypermelanose hat Auswirkungen auf das ästhetische Erscheinungsbild und lässt sich leider nur limitiert behandeln. Eine Pigmentlasertherapie ist aufgrund 


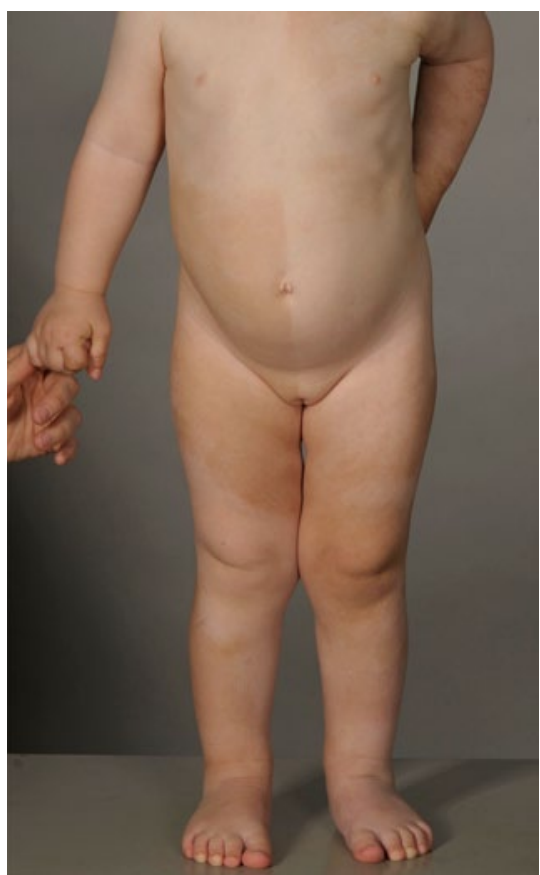

Abb. $7 \triangle$ Nävoide Hypermelanose als Manifestation eines kutanen Mosaiks in Schachbrettmuster-artiger Anordnung (mit freundl. Genehmigung L. Weibel)

der meist großen Ausdehnung schwierig durchführbar und von Rezidiven begleitet und die Dermabrasion zusätzlich von einem Vernarbungsrisiko.

\section{Weitere, nichtmelanozytäre kongenitale Nävi}

\section{Epitheliale Nävi}

Epitheliale Nävi entstehen durch Fehlbildungen des embryonalen (Neuro-)Ektoderms, aus dessen pluripotenten Zellen sich Keratinozyten und epidermale Anhangsorgane (Talgdrüsen, Schweißdrüsen, Haarfollikel) differenzieren [20].

Entsprechend dem vorherrschenden Gewebetyp unterscheidet man zwischen epidermalen Nävi im engeren Sinn (Keratinozytennävi) und den organoiden Nävi, die aus den epidermalen Anhangsorganen entstehen (z. B. Naevus sebaceus, „Talgdrüsennävus").

Keratinozytennävi zeigen kein eigentliches Entartungsrisiko, hingegen ist bei einzelnen Formen der organoiden Nävi mit Ausbildung von zumeist benignen, selten auch malignen Tumoren zu rechnen. Zudem können ausgedehnte epitheliale Nävi syndromale Assoziationen mit

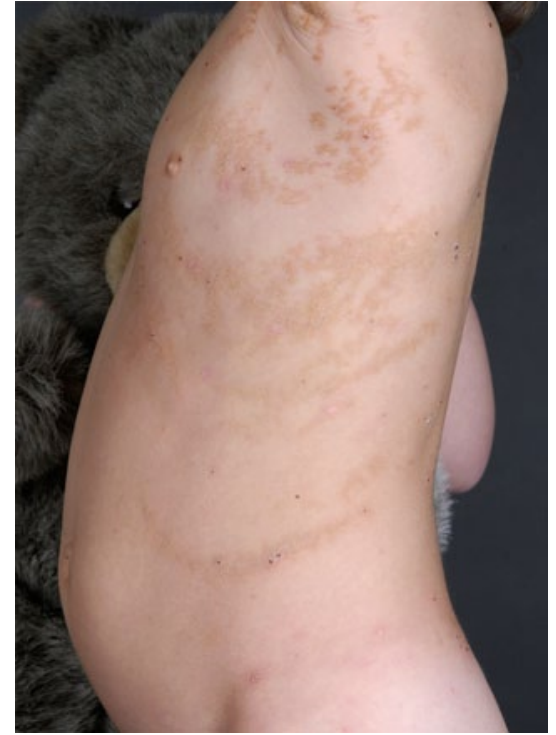

Abb. $8 \triangle$ Ausgedehnter epidermaler Nävus am Stamm in charakteristischer Blaschko-lineärer Anordnung

möglicher Manifestation im Bereich der meisten Organsysteme aufweisen.

\section{Epidermale Nävi (Keratinozytennävi)}

Sie sind meist bei Geburt vorhanden oder manifestieren sich im frühen Säuglingsalter. Es handelt sich um lineäre, entlang den Blaschko-Linien verlaufende, rötlichbräunliche hyperkeratotische Papeln und Plaques (• Abb. 8). In ca. einem Drittel der epidermalen Nävi kann eine Mutation im Fibroblasten-Wachstumsfaktorrezeptor (FGFR3) nachgewiesen werden [24]. Assoziierte Fehlbildungen in Form von neurologischen, muskuloskelettalen sowie ophthalmologischen Komplikationen können auftreten, vor allem in Assoziation mit FGF3-Mutationen. Daher ist bei ausgedehnten Läsionen eine neuropädiatrische sowie ophthalmologische Untersuchung sinnvoll, und es gilt, speziell auf das Längenwachstum der Kinder zu achten. Neben diesen unscharf definierten Assoziationen treten epidermale Nävi auch im Rahmen einiger gut definierter genetischer Syndrome auf, wie z. B. dem Proteus-Syndrom. Eine Spezialform der Keratinozytennävi stellt in etwa 5\% die epidermolytische Variante dar, die auf eine mosaikal vorliegende Keratin-1- oder -10-Mutation zurückzuführen ist. Falls diese die Keimbahn der betroffenen Person betrifft, kann bei deren Nachkommen das Vollbild einer keratinopathischen Ichthyose (bullöse kongenitale Ichthyose) auftreten.

Daneben ist die entzündliche Variante eines epidermalen Nävus gut bekannt und wird als ILVEN (,inflammatory linear verrucous epidermal nevus") bezeichnet. Letzterer tritt meist bereits im ersten Lebensjahr auf kann mit deutlichem Juckreiz einhergehen. Differenzialdiagnostisch ist an eine lineäre Psoriasis oder ein CHILD-Syndrom zu denken. Die Therapie des ILVEN ist oft frustran, so sind $\mathrm{CO}_{2}$-Laserablationen, Dermabrasion und Farbstofflaser mit Rezidiven behaftet. Kleinere oder schmale betroffene Areale können alternativ exzidiert werden.

\section{Organoide Nävi}

\section{Naevus sebaceus}

Es handelt sich um kongenital auftretende, solitäre, rundliche/ovaläre, haarlose, gelblich-orangefarbene flache Plaques meist im Bereich des Kopfes (• Abb. 9). Histologisch findet sich eine Vermehrung unreifer Talgdrüsen und undifferenzierter Haarfollikel [20]. Mit der Pubertät kommt es zu einer Stimulation der Talgdrüsen und konsekutiv zu einer meist störenden Dickenzunahme des Nävus mit verrukösem Umbau.

\section{》) Eine Totalexzision der Naevi sebacei im Vorschulalter ist empfehlenswert}

In 5-13\% entstehen auf dem Boden eines Naevus sebaceus Zweittumoren, die in der Regel allerdings benigne sind (Syringocystadenoma papilliferum, Trichoblastome; [25]). Letztere wurden früher häufig als Basalzellkarzinome fehldiagnostiziert. Dennoch sind selten auch echte Basalzellkarzinome möglich. Aus diesem Grund sowie vor allem aufgrund der ästhetisch störenden Entwicklung im Verlauf empfehlen wir prinzipiell eine Totalexzision der Naevi sebacei im Vorschulalter. $\mathrm{Zu}$ 


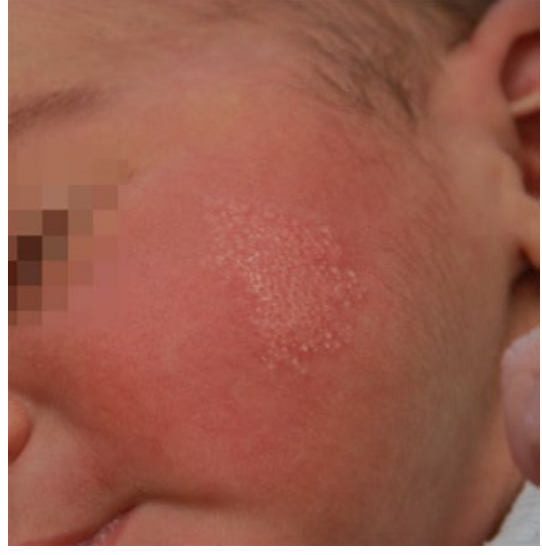

Abb. $9 \Delta$ Präsentation eines Naevus sebaceus an der Wange im Neugeborenenalter

diesem Zeitpunkt besteht noch eine erhöhte Hautelastizität, und die psychologische Ausgangslage für einen ambulanten Eingriff im Krankenhaus ist günstig. Bei ausgedehnten, bandförmigen Läsionen ist eine ophthalmologische und neuropädiatrische Untersuchung inklusive MRT des Schädels indiziert, um assoziierte Fehlbildungen im Sinne eines Naevus-sebaceus-Syndroms (Schimmelpenning-Feuerstein-Mims-Syndrom) auszuschließen. Bei diesem Syndrom finden sich ausgedehnte lineäre Naevi sebacei assoziiert mit neuroektodermalen Fehlbildungen (z. B. Hemimegalenzephalie, intrazerebrale Verkalkungen usw.), okulären Fehlbildungen (Iriskolobome usw.), Anfallsleiden, kontralateraler Hemiparese und Demenz [20].

\section{Schlussfolgerung}

Abschließend möchten wir zum Thema kongenitaler Nävi die Wichtigkeit eines multidisziplinären Managements dieser Patienten hervorheben. Allen voran sollten dabei im Kindesalter Vertreter der pädiatrischen Dermatologie, plastischen Kinderchirurgie und Kinderpsychologie beteiligt sein. Nur so ist es für die betroffenen Kinder und ihre Eltern möglich, sich breit informiert für operative Schritte zu entscheiden, deren Konsequenzen zu tragen und zuverlässig regelmäßigen Verlaufskontrollen nachzukommen. Eine Patientenvernetzung ist auch für Betroffene mit kongenitalen Nävi sehr wertvoll; für CMN stehen hier das deutschsprachi- ge Nävus-Netzwerk (http://www.naevusnetzwerk.de) sowie die weltweite, englischsprachige Organisation Nevus Outreach (http://www.nevus.org) zur Verfügung.

\section{Fazit für die Praxis}

- Das absolute Melanomrisiko von kleinen und mittelgroßen $\mathrm{CMN}$ beträgt $1 \%$ o bis $1 \%$ (Auftreten vor allem im späteren Erwachsenenalter).

- Das absolute Melanomrisiko von großen/riesigen CMN beträgt 1-10\% (Auftreten vor allem im frühen Kindesalter und bei einer Nävusgröße $>40 \mathrm{~cm}$ ).

- Die Durchführung einer MRT des Schädels (im Alter bis 6 Monate) ist indiziert bei CMN > 20 cm, multiplen Satelliten (>20 Stück) sowie bei mehreren mittelgroßen CMN.

- Die Indikation einer chirurgischen Therapie von CMN ist individuell zu evaluieren.

- Kleine und mittelgroße CMN mit Stigmatisierungspotenzial können gut im Kindesalter chirurgisch exzidiert werden.

- Die nävoide Hypermelanose ist eine nicht so seltene kutane Mosaikmanifestation, meist ohne assoziierte Fehlbildungen.

- Bei ausgedehnten epidermalen Nävi ist eine neuropädiatrische sowie ophthalmologische Untersuchung sinnvoll, und es ist auf Skelett-/Wachstumsauffälligkeiten zu achten.

- In Naevi sebacei entstehen in 5-13\% meist benigne Zweittumoren, auch präpubertär. Eine Exzision ist prinzipiell auch aus ästhetischer Indikation zu empfehlen, idealerweise im Vorschulalter.

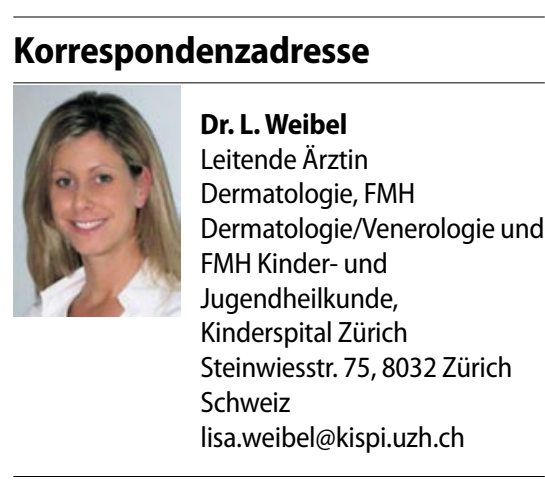

Interessenkonflikt. Der korrespondierende Autor gibt für sich und seine Koautoren an, dass kein Interessenkonflikt besteht.

\section{Literatur}

1. Castilla EE, Graca Dutra M da, Orioli-Parreiras IM (1981) Epidemiology of congenital pigmented naevi: I. Incidence rates and relative frequencies. $\mathrm{Br}$ J Dermatol 104(3):307-315

2. Krengel S, Scope A, Dusza SW et al (2012) New recommendations for the categorization of cutaneous features of congenital melanocytic nevi. J Am Acad Dermatol [Epub ahead of print]

3. Marghoob AA, Schoenbach SP, Kopf AW et al (1996) Large congenital melanocytic nevi and the risk for the development of malignant melanoma. A prospective study. Arch Dermatol 132(2):170175

4. Krengel S (2012) New aspects of congenital melanocytic nevi. Hautarzt 63(2):82-88

5. Bauer J, Curtin JA, Pinkel D, Bastian BC (2007) Congenital melanocytic nevi frequently harbor NRAS mutations but no BRAF mutations. J Invest Dermatol 127(1):179-182

6. Krengel $\mathrm{S}$, Breuninger $\mathrm{H}$, Beckwith $\mathrm{M}$, Etchevers $\mathrm{HC}$ (2011) Meeting report from the 2011 International Expert Meeting on Large Congenital Melanocytic Nevi and Neurocutaneous Melanocytosis, Tübingen. Pigment Cell Melanoma Res 24(4):E1-E6

7. Shakhova O, Zingg D, Schaefer SM et al (2012) Sox 10 promotes the formation and maintenance of giant congenital naevi and melanoma. Nat Cell Biol 14(8):882-890

8. Kinsler V, Shaw AC, Merks JH, Hennekam RC (2012) The face in congenital melanocytic nevus syndrome. Am J Med Genetics 158A(5):1014-1019

9. Kinsler VA, Abu-Amero S, Budd P et al (2012) J Invest Dermatol 132(8):2026-2032

10. Slutsky JB, Barr JM, Femia AN, Marghoob AA (2010) Large congenital melanocytic nevi: associated risks and management considerations. Semin Cutan Med Surg 29(2):79-84

11. Krengel S, Hauschild A, Schäfer T (2006) Melanoma risk in congenital melanocytic naevi: a systematic review. Br J Dermatol 155(1):1-8

12. Berg $P$, Lindelöf $B$ (2003) Congenital melanocytic naevi and cutaneous melanoma. Melanoma Res 13(5):441-445

13. Kinsler VA, Chong WK, Aylett SE, Atherton DJ (2008) Complications of congenital melanocytic naevi in children: analysis of 16 year's experience and clinical practice. Br J Dermatol 159(4):907-914

14. Bett BJ (2006) Large or multiple congenital melanocytic nevi: occurrence of neurocutaneous melanocytosis in 1008 persons. J Am Acad Dermatol 54(5):767-777 
15. Kinsler V, Bulstrode N (2009) The role of surgery in the management of congenital melanocytic naevi in children: a perspective from Great Ormond Street Hospital. J Plast Reconstr Surg 62(5):595601

16. Masnari O, Landolt MA, Roessler J et al (2012) Self- and parent-perceived stigmatisation in children and adolescents with congenital or acquired facial differences. J Plast Reconstr Aesthet Surg 65(12):1664-1670

17. Masnari O, Schiestl C, Rössler J et al (2012) Stigmatization predicts psychological adjustment and quality of life in children and adolescents with a facial difference. J Pediatr Psychol [Epub ahead of print]

18. Hausschild A, Garbe C, Bauer J et al (2006) Melanocytic nevi. JDDG 4(8):686-697
19. Schaffer JV, Orlow SJ, Lazova R, Bolognia JL (2001) Speckled lentiginous nevus-classic congenital melanocytic nevus hybrid not the result of "collision". Arch Dermatol 137(12):1655

20. Höger PH (Hrsg) (2011) Kinderdermatologie. Differenzialdiagnose und Therapie bei Kindern und Jugendlichen. F. Schattauer, Stuttgart

21. Sekar S, Kuruvila M, Pai HS (2008) Nevus of Ota: a series of 15 cases. Indian J Dermatol Venereol Leprol 74(2):125-127

22. Di Lernia V (2007) Linear and whorled hypermelanosis. Pediatr Dermatol 24(3):205-210

23. Nehal KS, PeBenito R, Orlow SJ (1996) Analysis of 54 cases of hypopigmentation and hyperpigmentation along the lines of Blaschko. Arch Dermatol 132(10):1167-1170
24. Hafner C, Oers JM van, Vogt T et al (2006) Mosaicism of activating FGFR3 mutations in human skin causes epidermal nevi. J Clin Invest 116(8):22012207

25. Rosen H, Schmidt B, Lam HP et al (2009) Management of nevus sebaceous and the risk of basal cell carcinoma: an 18-year review. Pediatr Dermatol 26(6):676-681

\section{Hier steht eine Anzeige.}

\section{Springer}

